\title{
Evaluation of the Crystal Rotation Behavior at the Fatigue Crack Tip in Pure Titanium Films*
}

\author{
Kenichi SHIMIZU**, Tashiyuki TORII** and Yasuto FUJINO*** \\ ** Okayama University, 3-1-1, Tsushima-naka, Okayama, 700-8530, Japan \\ E-mail: kshimizu@mech.okayama-u.ac.jp \\ *** Mitsubishi Heavy Industries, LTD.
}

\begin{abstract}
Using a fatigue testing method by which fatigue cracks can be initiated and propagated in a film adhered to cover an elliptical through-hole in a base plate subjected to push-pull cyclic loads, annealed pure titanium films with the thickness of $90 \mu \mathrm{m}$ were fatigued under a constant stress amplitude with a stress ratio of -1 . The crystal rotation behavior with the tensile/compressive loading at the fatigue crack tip was evaluated from the crystal orientation measured by EBSD (Electron Back-scatter Diffraction) method. The titanium films under the tensile /compressive stress condition were prepared as follows; the films were adhered to the base plates loaded by a servo hydraulic testing machine, then the base plates were detached from the testing machine. From the crystal rotation behavior at the fatigue crack tip, it was found that the crystal rotation angle was relatively small and the rotation axis tended to different directions between the upper side and lower side of the grain in front of the crack tip. The fatigue crack propagated along the borderline between two regions in the crystal grain where the crystal rotation axes with different directions were obtained before the fatigue crack propagation.
\end{abstract}

Key words: Titanium Film, Fatigue Crack Tip, Crystal Rotation, EBSD Method

\section{Introduction}

The physical qualities such as high strength, toughness, durability, low density, corrosion resistance and biological compatibility make titanium useful in a wide range of structural, chemical, petrochemical, marine and biomaterial applications. For reliability of products made from titanium, it is necessary to estimate the fatigue properties of the titanium. Since the pure titanium with a hexagonal closed packed (hcp) crystalline structure have less slip systems as compared to a body centered cubic (bcc) and a face centered cubic (fcc), the microstructures such as crystal orientations and grain boundaries seem to influence remarkably to the fatigue crack propagation behavior, especially used in the film form with one or a few crystal grains along the thickness. However, as fatigue testing presents a serious difficulty with regard to gripping small specimens, the fatigue properties of the film specimen have been hardly discussed as compared with those of the bulk specimen.

In this study, a film fatigue testing method was proposed by which fatigue crack initiation and propagation occurred on a film bonded to a circular through-hole in a base plate subjected to push-pull cyclic loads ${ }^{(1),(2)}$. If a film adhered to a through-hole in a base plate is regarded as the ellipsoidal inclusion in Eshelby's model ${ }^{(3)}$, the strain and the stress will be uniform in the film. In this way, it is possible to conduct film fatigue testing by stress cycling on the base plate. Using this fatigue testing method of films, fatigue 
properties of the annealed titanium film with the thickness of $90 \mu \mathrm{m}$ were examined. Then, the static tensile/compressive stress was loaded to the fatigued film specimen in the scanning electron microscope (SEM) chamber and the crystal rotation behavior at the fatigue crack tip was investigated by measuring the crystal orientation obtained from electron backscatter diffraction (EBSD) method ${ }^{(4),(5)}$. Subsequently, the fatigue crack was propagated in the area analyzed by the EBSD method and the relationship between the crystal rotation in front of the crack tip and the crack propagation was discussed for the titanium films.

\section{Experimental Procedure}

\subsection{Specimens}

The pure titanium films with a thickness of $t_{\mathrm{f}}=90 \mu \mathrm{m}$ were annealed at $1073 \mathrm{~K}$ for one hour in a vacuum furnace. The base specimens of high-tensile steel (HT60) were machined to the dimension with a circular through-hole shown in Fig. 1, then polished with emery paper and finally annealed at $873 \mathrm{~K}$ for one hour in a vacuum furnace. A base plate with smooth surfaces for both sides was defined as the base plate "A" and that with a dent on one side was defined as the base plate "B". The thickness of both base plates was $t_{\mathrm{b}}=1.0 \mathrm{~mm}$. The film specimen was cut into a $30 \times 30 \mathrm{~mm}^{2}$ rectangle and a through-hole of $0.5 \mathrm{~mm}$ diameter was made at the film center by using a drilling machine. The film was bonded to the base plate " $\mathrm{B}$ " so that the rolling direction was parallel to the loading direction of the base plate as illustrated in Fig. 1. Finally, the base plate "A" was bonded to the film adhered to the base plate " $\mathrm{B}$ ".

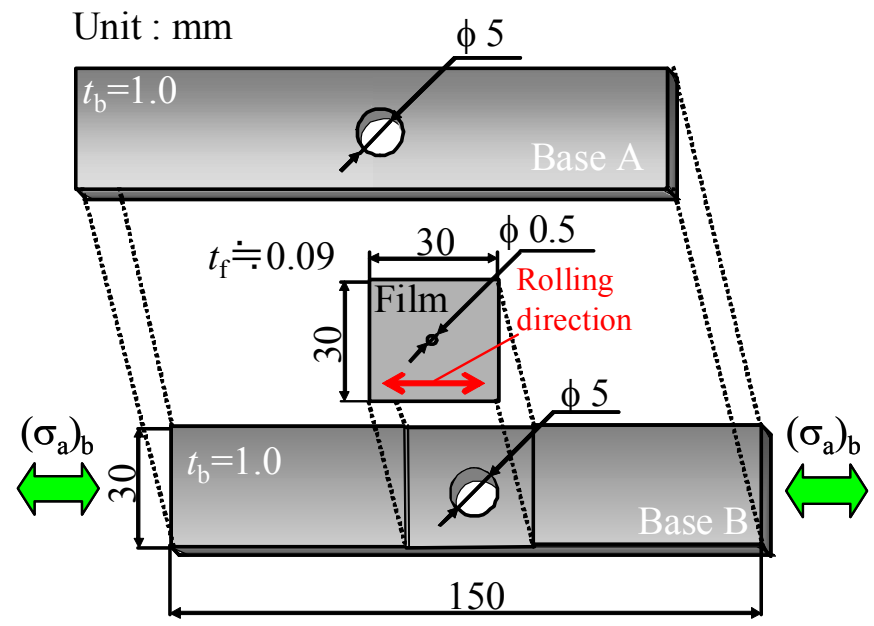

Fig. 1 Dimension of the film fatigue specimen.

\subsection{Film Fatigue Testing}

The film was fatigued in accordance with the displacement along the hole circumference in the base plates subjected to push-pull sinusoidal cyclic loads with a constant stress amplitude, $\left(\sigma_{\mathrm{a}}\right)_{\mathrm{b}}=90 \mathrm{MPa}$, at a speed of $20 \mathrm{~Hz}$ and a stress ratio of $R=-1$, using a servo-hydraulic fatigue testing machine. In this fatigue testing, the cyclic strains and stresses are uniform in the film adhered to a through-hole in a base plate subjected to cyclic loading, as described in the ellipsoidal inclusion in Eshelby's model ${ }^{(3)}$. The stress distribution calculated using a three-dimensional elastic FEM analysis was almost uniform on the film. The value of the stress was almost 1.6 times of applied stress $\left(\sigma_{\mathrm{a}}\right)_{\mathrm{b}}$. The fatigue crack propagation behavior on the film was observed using an optical microscope attached to the testing machine. 


\subsection{Crystal Orientation Analysis under the Static Tensile/Compressive Loading}

The crystal orientation of titanium films was analyzed using an EBSD system (Link OPAL, Oxford Instruments) ${ }^{(4)}$. The analyzed area was $128 \times 168 \mu \mathrm{m}$ with the measured points of $100 \times 128=12800$.

EBSD measurement under the static tensile loading was experimented as follows; the film with the fatigue crack was detached from the base plates " $\mathrm{A}$ " and " $\mathrm{B}$ " by ultrasonic cleaning in acetone. Then, the base plate " $\mathrm{B}$ " was compressed at $\sigma_{b}=90 \mathrm{MPa}$ using the servo-hydraulic fatigue testing machine and the fatigued film was bonded to the compressed base plate "B" as indicated in Fig. 2. When the base plate "B" was unloaded after a couple of hours, the film was tensed under the same conditions at the maximum stress of the cyclic loading. Similarly, the compressed film could be obtained by bonding it to the tensed base plate " $\mathrm{B}$ ". The crystal orientation of titanium films bonded to the base plate " $\mathrm{B}$ " was able to be measured in the SEM chamber by EBSD method.

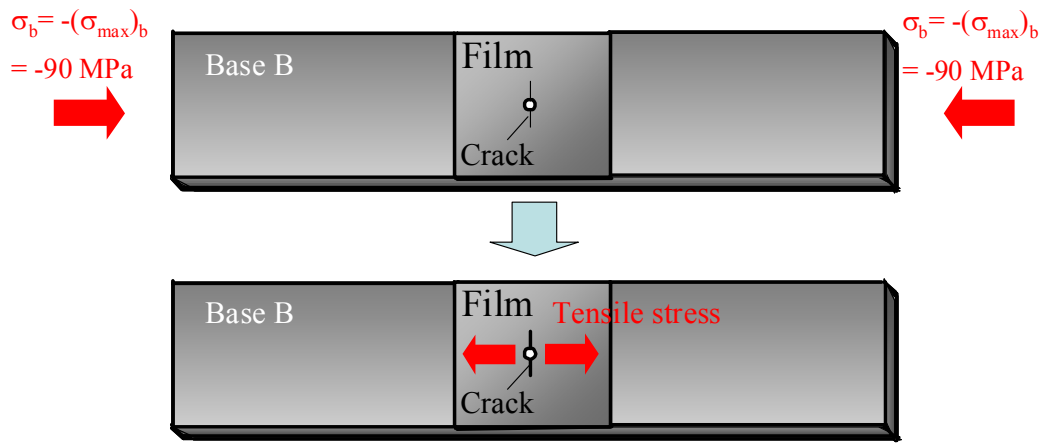

Fig. 2 Method of the preparing the specimen with the tensile stress.

\subsection{Calculation of Crystal Rotation Angle}

Figure 3 illustrates the relationship between the crystal axes and the specimen axes. The cosines of $\alpha_{1}, \beta_{1}$ and $\gamma_{1}$ are the angles between [1 100$]$ and rolling direction, [1 100$]$ and transverse direction and [1 100$]$ and normal direction respectively. They form the first row of the orientation matrix, $A$, as shown in Eq. (1). The other two rows are formed in a similar manner from the cosines of angles between [11 20$]$ and rolling direction, [11 $\overline{2} 0]$ and transverse direction and [1120] and normal direction; then [0001] and rolling direction, [0001] and transverse direction and [0001] and normal direction. These angles are $\alpha_{2}, \beta_{2}$, $g_{2}$ and $\alpha_{3}, \beta_{3}, \gamma_{3}$ respectively. For clarity only $\alpha_{1}, \beta_{1}, \gamma_{1}$ are shown on Fig. 3.

$$
A=\left(\begin{array}{ccc}
\cos \alpha_{1} & \cos \beta_{1} & \cos \gamma_{1} \\
\cos \alpha_{2} & \cos \beta_{2} & \cos \gamma_{2} \\
\cos \alpha_{3} & \cos \beta_{3} & \cos \gamma_{3}
\end{array}\right)
$$

When an orientation matrix obtained from a same point under unloaded and loaded conditions are defined as $A_{2}$ and $A_{1}$ respectively, the crystal rotation angle, $\theta$, can be calculated as follows ${ }^{(6),(7)}$ :

$$
\begin{aligned}
& M_{21}=A_{2}^{-1} A_{1} \\
& \cos \theta=\frac{\operatorname{Tr} M_{21}-1}{2}
\end{aligned}
$$

where $M_{21}$ is the matrix which represents the rotation of $A_{1}$ onto $A_{2}, \operatorname{Tr} M_{21}$ is the summation of the diagonal elements. 
When there is a possibility that the direction of the film changes at the debonding/bonding process to the base plate, the similar rotation angles and the axes are calculated on the whole area owing to a rigid body rotation behavior. The effect of the rigid body rotation is able to be eliminated by the inverse rotation.

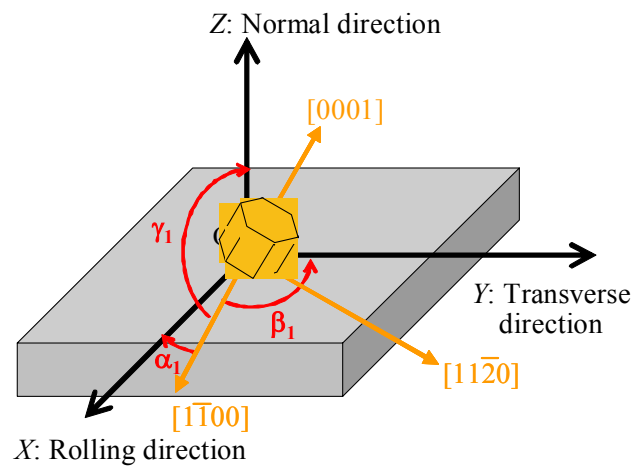

Fig. 3 Definition of specimen axes and crystal axes.

\section{Experimental Results}

\subsection{Crystal Orientation}

Figure 4 shows the crystal orientation map (COM) measured from the cross section of the titanium film and Fig. 5 shows the color key used to produce COM. Number of grains per thickness of $t_{\mathrm{f}}=100 \mu \mathrm{m}$ is one or two and a [0001] texture can be observed in the COM showing the orientation to the normal direction.

A scanning electron micrograph around the fatigue crack is shown in Fig. 6 and the crystal orientation map to the rolling direction under the unloaded condition is expressed in Fig. 7. It is shown that the grain size is about $70 \mu \mathrm{m}$.

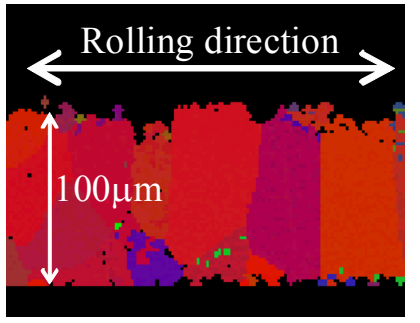

(a) Orientation to the normal direction.

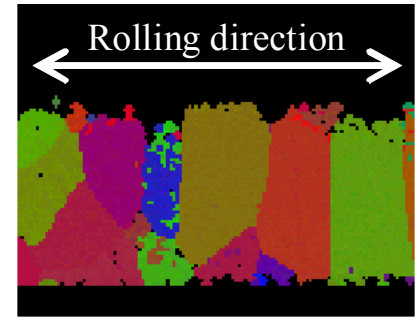

(b) Orientation to the transverse direction.

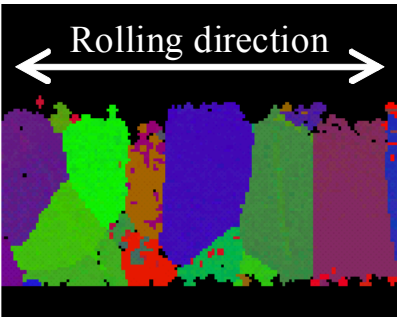

(c) Orientation to the rolling direction.

Fig. 4 Crystal orientation maps observed from the cross section of the film.

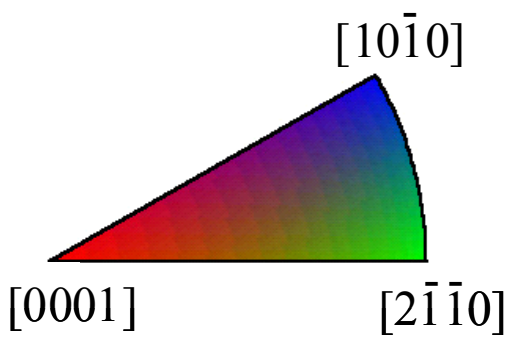

Fig. 5 Color key. 


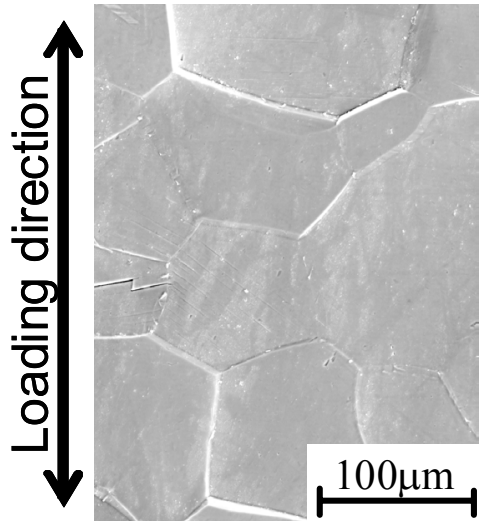

Fig. 6 Scanning electron micrograph of the fatigue crack tip under the unloaded condition.

(before fatigue crack propagation)

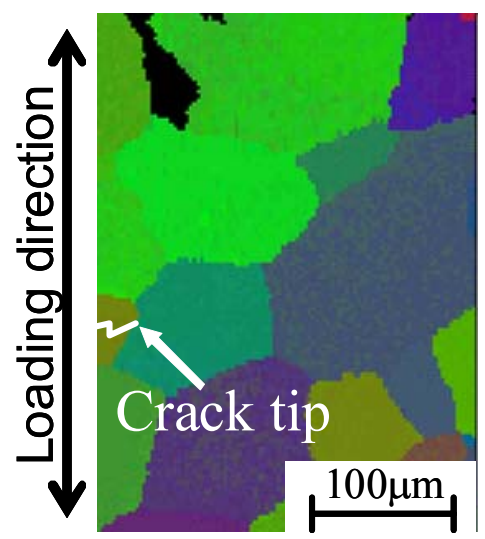

Fig. 7 COM to the rolling direction under the unloaded condition. (before fatigue crack propagation)

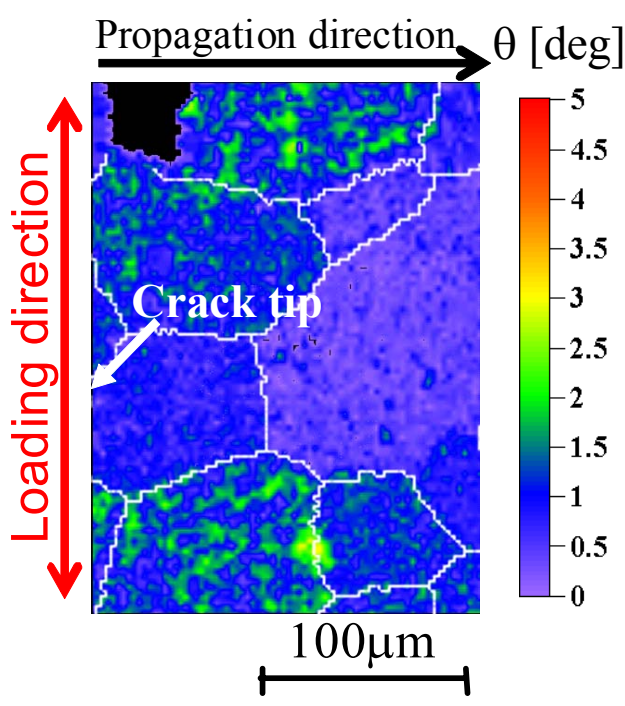

(a) Rotation angle.

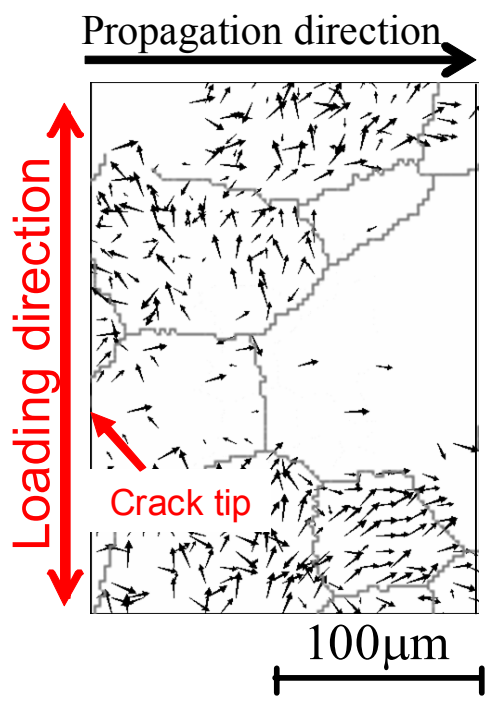

(b) Rotation axis.

Fig. 8 Crystal rotation behavior with the tensile loading $\left(\sigma_{\mathrm{b}}=0 \mathrm{MPa} \rightarrow 90 \mathrm{MPa}\right)$.
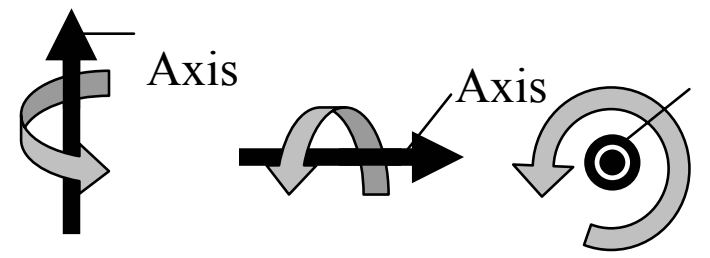

Axis

Fig. 9 Relationship between the rotation axis and the rotation direction.

\subsection{Crystal Rotation with Static Loading}

Figure 8 (a) shows the distribution of the crystal rotation angle, $\theta$ with the static tensile loading, $\sigma_{b}=0 \mathrm{MPa} \rightarrow 90 \mathrm{MPa}$. The area illustrated by black shows the region in which the crystal rotation angle is not able to be calculated for the severe damage on the film surface. The large value of crystal rotation angle is evaluated relatively far from the fatigue crack tip 
and the area with little rotation angle can be seen in front of the crack tip. The distribution of the crystal rotation axis at the point where the rotation angle is 0.8 degree and over is shown in Fig. 8(b). White lines in Fig. 8(a) and gray lines in Fig. 8(b) indicate the grain boundary on which the evaluated misorientaion angle is 5.0 degree and over in Fig. 7. Figure 9 shows the explanation about the appearance of the rotation axis observed from the normal direction. The rotation direction around the axis is clockwise to the arrow. As the rotation axis is a unit vector, the short axis observed from the normal direction tends to the normal direction to the surface. The rotation axes are found to tend to similar directions in each grain as shown in Fig. 8(b).

Figure 10 shows the distribution of the crystal rotation angle, $\theta$, and that of the rotation axis with the rotation angle of 0.8 degree and over under the compressive loading process, $\sigma_{\mathrm{b}}=90 \mathrm{MPa} \rightarrow-90 \mathrm{MPa}$. While the small value of rotation angle is evaluated in front of the crack tip similarly to the tensile loading process as indicated in Fig. 8, the value of crystal rotation angle is larger in the compressive process than in the tensile process owing to the twofold change of the load. The rotation axes tend to similar directions in each grain except for the grain in front of the fatigue crack tip as illustrated by the bold line where the axis directions are different between the upper side and lower side of the crystal grain.

Figure 11 shows the fatigue crack propagated in the area where the crystal orientation is measured by the EBSD method. From the relationship between the crack propagation path and the grain boundary as illustrated in Fig. 12, the transgranular crack was observed in the titanium film. The crack propagation rate is about $5 \times 10^{-9} \mathrm{~m} /$ cycle in the area.

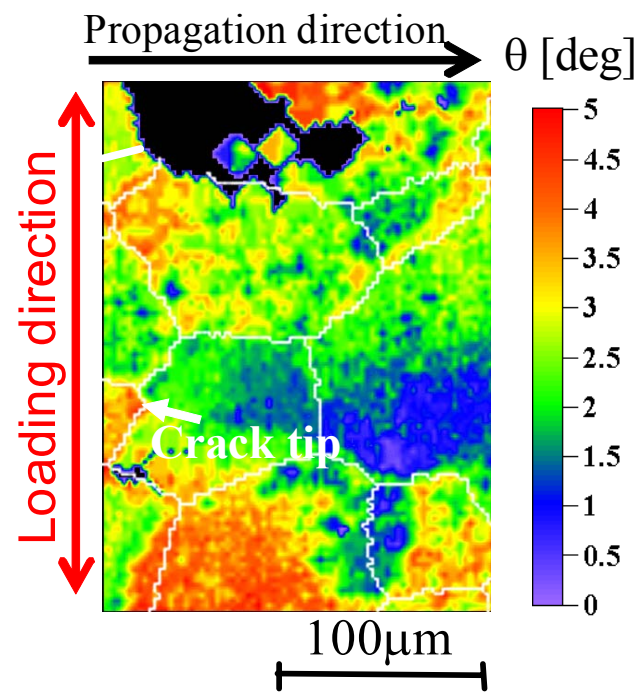

(a) Rotation angle.

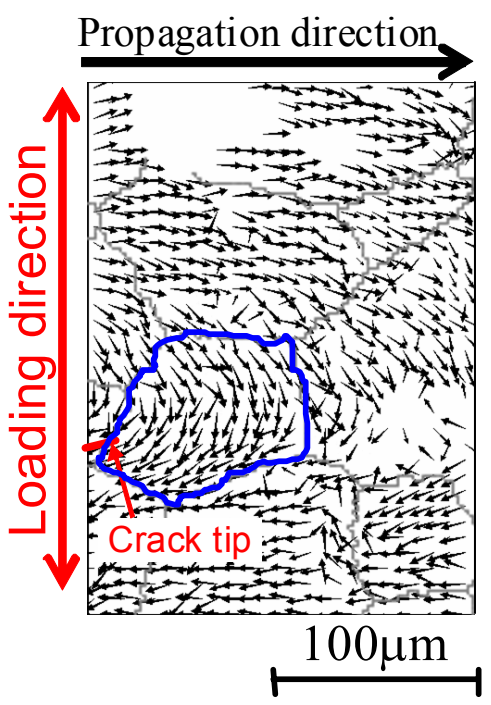

(b) Rotation axis

Fig. 10 Crystal rotation behavior with the compressive loading $\left(\sigma_{b}=90 \mathrm{MPa} \rightarrow-90 \mathrm{MPa}\right)$.

\section{Discussion}

Figure 13 shows the magnification of the area surrounded by the white dotted line as shown in Fig. 11. As the fatigue crack runs parallel with the slip lines, it is considered that the crack propagates along the slip line initiated in front of the crack tip. From the relationship between the angle of the slip line and the crystal orientation obtained by the EBSD method, the slip plane worked in the hcp crystalline structure is able to be determined a prismatic slip plane as shown in Fig. 14. The crystal rotation axes evaluated at the compressive process are overlayed on the magnification of the fatigue crack tip and the crack path obtained after the fatigue crack propagation is expressed by the bold line in 
Fig. 14. A remarkable change of the axis direction is observed on the bold line as a borderline between the upper side and the lower side of the crack path because the deformations on the upper side and lower side of the crystal grain seem to be different under the influence of the surrounding crystal orientation. As a result, it is possible that slip planes separate on the borderline owing to the different direction of the crystal rotation as illustrated by the hep crystalline structures. Although the relationship between the slip direction and the crystal rotation direction must be discussed furthermore, there is a possibility that the crack propagation path is predicted by evaluating the crystal rotation with the static loading in front of the fatigue crack tip.

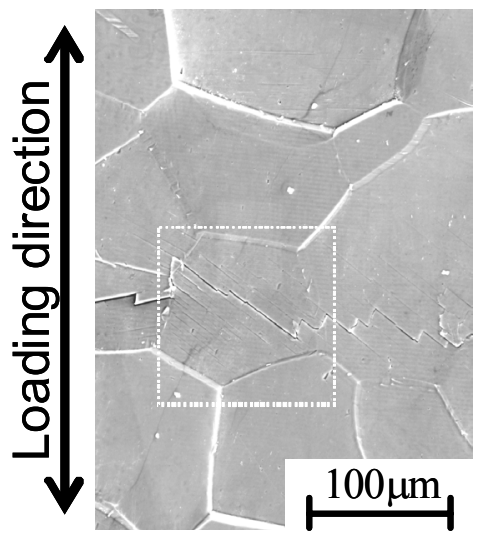

Fig. 11 Scanning electron micrograph around the fatigue crack. (after fatigue crack propagation)

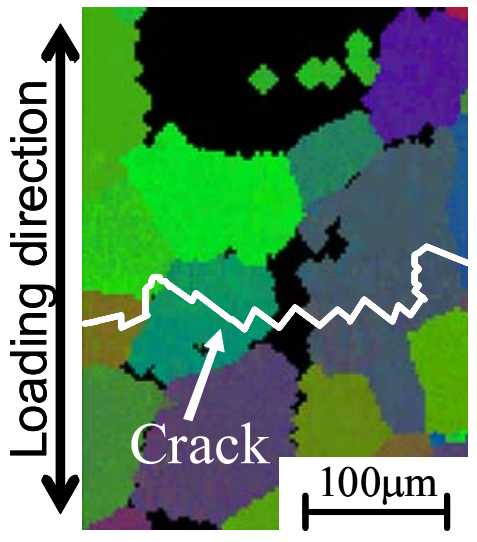

Fig. 12 COM to the rolling direction.

(after fatigue crack propagation)

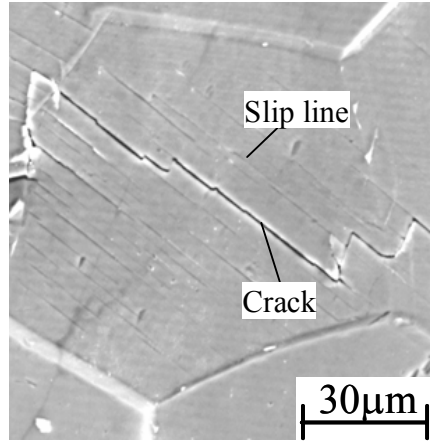

Fig. 13 Magnification of the fatigue crack and slip lines.

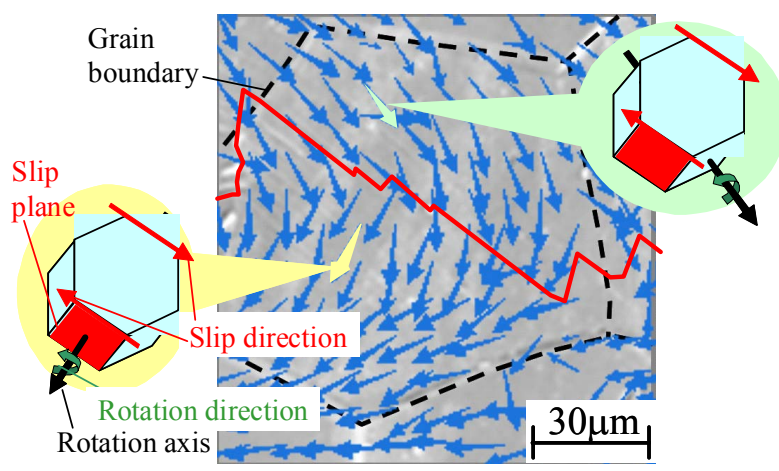

Fig. 14 Schematic figure of the hcp crystalline structure rotation.

\section{Conclusions}

A pure titanium film with the thickness of $90 \mu \mathrm{m}$ was fatigued and a crystal rotation behavior with the tensile/compressive loading at the fatigue crack tip was evaluated by measuring the crystal orientation using EBSD method. The main results obtained are as follows.

(1) The crystal rotation angle and the rotation axis were evaluated at the fatigue crack tip under the tensile and the compressive loaded conditions. As a result, the small value of rotation angle is evaluated and the axis directions are different between the upper side and 
lower side of the crystal grain in front of the crack tip.

(2) From the relationship between the angle of the slip line and the crystal orientation obtained by the EBSD method, the slip plane worked in the hep crystalline structure was able to be determined. The fatigue crack propagates along the slip line initiated in front of the crack tip in the titanium film.

(3) The crack path obtained after fatigue testing traced the line on which the rotation axis directions are different between the upper side and lower side in the crystal grain. There is a possibility that the crack propagation path is predicted by evaluating the crystal rotation behavior with the static loading in front of the fatigue crack.

\section{References}

(1) Torii, T. et al., JSME Int. J., Ser. A, Vol. 39-1 (1996), pp. 34-41.

(2) Torii, T. et al., Proceedings of Advances in Electronic Packaging 1999, ASME, Vol. 1 (1999), pp. 867-874.

(3) Mura, T., Micromechanics of Defects in Solids, Martinus Nijhoff Publishers, (1982).

(4) Randle, V., Microtexture Determination and its Applications, The Institute of Materials, (1992).

(5) Shimizu, K. et al., Fatigue \& Fracture of Engineering Materials \& Structures, Vol. 28 (2005), pp.221-227.

(6) Shimizu, K. et al., Proceedings of 2005 ASME/Pacific Rim Technical Conference and Exhibition on Integration and Packaging of MEMS, NEMS, and Electronic Systems (InterPACK 2005), (2005), IPACK2005-73134.

(7) Shimizu, K. et al., Proceedings of ATEM '07 (International Conference on Advanced Technology in Experimental Mechanics 2007), (2007), OS05-1-6. 